\title{
Article \\ Epidermal Barrier Function and Skin Homeostasis in Skin with Permanent and Adhesive Tattoos: A Cross-Sectional Study
}

\author{
Jose-Pablo Serrano-Serra ${ }^{1}$, Trinidad Montero-Vilchez ${ }^{2,3, * \mathbb{C}}$, Agustin Buendia-Eisman ${ }^{1}$ \\ and Salvador Arias-Santiago 1,2,3 \\ 1 Dermatology Department, Faculty of Medicine, University of Granada, 18012 Granada, Spain; \\ joserranoserra@gmail.com or joserranoserra@correo.ugr.es (J.-P.S.-S.); abuendia@ugr.es (A.B.-E.); \\ salvadorarias@ugr.es (S.A.-S.) \\ 2 Dermatology Department, Hospital Universitario Virgen de las Nieves, 18012 Granada, Spain \\ 3 Instituto de Investigación Biosanitaria GRANADA, 18012 Granada, Spain \\ * Correspondence: tmonterov@gmail.com or tmonterov@correo.ugr.es; Tel.: +34-958023259
}

Citation: Serrano-Serra, J.-P.;

Montero-Vilchez, T.; Buendia-Eisman,

A.; Arias-Santiago, S. Epidermal

Barrier Function and Skin Homeostasis in Skin with Permanent and Adhesive Tattoos: A Cross-Sectional Study. J. Clin. Med. 2021, 10, 888. https:// doi.org/10.3390/jcm10040888

Academic Editor: Masutaka Furue

Received: 22 January 2021

Accepted: 14 February 2021

Published: 22 February 2021

Publisher's Note: MDPI stays neutral with regard to jurisdictional claims in published maps and institutional affiliations.

Copyright: (C) 2021 by the authors. Licensee MDPI, Basel, Switzerland. This article is an open access article distributed under the terms and conditions of the Creative Commons Attribution (CC BY) license (https:/ / creativecommons.org/licenses/by/ $4.0 /)$.

\begin{abstract}
Tattoos are a current trend, but their impact on skin homeostasis and epidermal barrier function is not well known. So, the aims of this study are (1) to investigate epidermal barrier function and skin homeostasis in skin with permanent tattoos, adhesive temporary tattoos and non-tattooed skin, and (2) to analyze the effect of petrolatum on skin with permanent and adhesive tattoos. In total, 67 tattoos were enrolled ( 34 permanent tattoos and 33 adhesive tattoos). Temperature, transepidermal water loss (TEWL), stratum corneum hydration (SCH), erythema and total antioxidant capacity (TAC) were measured in skin with permanent tattoos, adhesive tattoos and non-tattooed skin before and after petrolatum application. The temperature was lower $\left(30.47^{\circ} \mathrm{C}\right.$ vs. $\left.31.01{ }^{\circ} \mathrm{C} ; p=0.001\right)$ on skin with permanent tattoos than non-tattooed skin, while SCH (48.24 Arbitrary Units (AU) vs. 44.15 AU; $p=0.008)$ was higher. Skin with adhesive tattoos showed lower temperature, SCH (21.19 AU vs. 41.31 AU; $p<0.001)$ and TAC (1.27 microcoulombs (uC) vs. $3.48 \mathrm{uC} ; p<0.001)$, and higher TEWL $\left(8.65 \mathrm{~g} / \mathrm{h} / \mathrm{m}^{2}\right.$ vs. $\left.6.99 \mathrm{~g} / \mathrm{h} / \mathrm{m}^{2} ; p=0.003\right)$, than non-tattooed skin. After petrolatum application, the temperature decreased on skin with permanent tattoos, and TEWL and SCH decreased on skin with adhesive tattoos. Adhesive tattoos may affect skin barrier function, while permanent tattoos may have a lower impact. Tattooed and non-tattooed skin responds in different ways to moisturizers.
\end{abstract}

Keywords: adhesives; antioxidants; epidermis; petrolatum; skin barrier; tattooing

\section{Introduction}

Tattoos have been present in various cultures throughout history, mainly in relation to specific groups or linked to certain mystical or religious practices [1]. Nowadays, tattoos have spread to wider social circles and their popularity has increased notably. Recently, Kluger et al. observed that the prevalence of tattooed people ranged from $11.70 \%$ to $31.50 \%$ depending on the country. Tattoos were more frequent in women and young people [2]. Furthermore, the prevalence of tattoos in Europe is increasing. Since 2003, Borkenhagen et al. have observed an increase in the tattooed population in Germany [3]. Kluger et al. also showed higher rates of tattooed people in France from 2010 to 2017 [4,5].

The tattooing process consists of puncturing the skin with fine mechanized needles, at a speed of 50 to 3000 times per minute. Puncture depth may vary between 1 and $4 \mathrm{~mm}$ [6]. Approximately $1 \mathrm{mg} / \mathrm{cm}^{2}$ of pigment will remain on the skin [7]. Once ink particles enter the dermis, they are ingested by macrophages [8,9]. They may then disseminate systemically throughout the body, reaching the lymph nodes or the liver $[10,11]$.

The dyes used in the tattooing process are predominantly insoluble or semi-soluble pigments, to which water and other compounds are added. Black pigments are derived from soot and are designed for industrial use. Some dyes contain a large number of organic 
pigments, which are used in association with metals to obtain the ink composition. Some pigments have not been toxicologically tested for use on humans [7].

The rate of side effects associated with tattoos is around $2 \%$, the most frequent being infectious, allergic and granulomatous complications [12]. The potential carcinogenic effects of tattoos is controversial [13]. Moreover, tattoos can hinder the early diagnosis of pigmented skin lesions [14].

Sticker or adhesive tattoos are a very popular kind of tattoo, especially among children, who are the most vulnerable group to suffer from the secondary effects of chemicals [15]. The main ingredients of the ink are colorants, although detailed information about their composition is usually unavailable. Potentially harmful substances have been found in these products, such as aromatic amines, heavy metals and adhesive substances, which may cause several side effects, such as skin irritation [16].

There is little information regarding the effect of tattoos on skin barrier function and skin homeostasis. It has been noted that tattoos decrease the sweat rate and increase sweat sodium concentration [6]. Furthermore, it has been observed that skin capacitance is higher in tattooed skin compared to non-tattooed skin [17]. Due to the increase in the number of people with tattoos and the lack of awareness about the repercussions of tattooing, research on the effect of tattoos on the skin is increasingly relevant.

Differences in the skin barrier function parameters measured in tattooed and nontattooed skin are expected because of the skin damage inflicted by tattoos. The objective of this study was to compare the parameters of cutaneous homeostasis and epidermal barrier function (temperature, transepidermal water loss (TEWL), stratum corneum hydration $(\mathrm{SCH})$, erythema and total antioxidant capacity (TAC)) in skin with permanent tattoos and temporary adhesive tattoos, compared to non-tattooed skin. It was also evaluated if tattooed and non-tattooed skin responds in different ways to moisturizer application.

\section{Materials and Methods}

2.1. Design

(A) A cross-sectional study to assess skin homeostasis differences between nontattooed skin, skin with permanent tattoos and skin with adhesive tattoos.

(B) Before and after study in patients with tattoos to assess changes in skin homeostasis after the application of a moisturizer (petrolatum).

\subsection{Study Population}

Participants were recruited from January 2020 to March 2020 at the Dermatology Department of the Hospital Universitario Virgen de las Nieves in Granada. Patients who had a tattoo were invited to participate. They were people affected by skin diseases not associated with their tattoos. People who accompanied patients and other voluntary participants were also recruited.

- Inclusion criteria: People over 18 years old, who had got their tattoo more than one month ago (in the case of permanent tattoos), without a skin reaction during the evaluation and who signed the informed consent form.

- Exclusion criteria: People who did not sign the informed consent form or who presented a skin disorder associated with the tattoo, or any cutaneous active (with an outbreak in the previous five years) inflammatory disease (psoriasis, atopic dermatitis).

\subsection{Study Variables Measurement}

Homeostasis parameters related to epidermal barrier function were measured. $\mathrm{SCH}$ (in arbitrary units, using Corneometer ${ }^{\circledR} \mathrm{CM} 825$, Mirocaya, Bilbao, Spain), TEWL (in $\mathrm{g} \cdot \mathrm{m}^{-2} \mathrm{~h}^{-1}$, using Tewameter ${ }^{\circledR}$ TM 300, Mirocaya, Bilbao, Spain), erythema index (in arbitrary units, using Mexameter ${ }^{\circledR}$ MX 18, Mirocaya, Bilbao, Spain) and skin temperature (in ${ }^{\circ} \mathrm{C}$, using Skin-Thermometer ST 500, Mirocaya, Bilbao, Spain) were measured by a Multi Probe Adapter (MPA, Courage + Khazaka electronic GmbH, Mirocaya, Bilbao, Spain). Total antioxidant capacity (TAC) was measured using a conductive hydrogel placed on the skin, 
applying an electrochemical method, with the $\mathrm{eBQC}^{\circledR}$ device (Bioquochem S.L. (BQCkit), Madrid, Spain), and expressed in microcoulombs. TAC is divided into two sections: fast antioxidants (Q1) have lower oxidation potential than slow antioxidants (Q2) [18]. Antioxidant capacity parameters were measured once and the other parameters were measured ten times, their average being used for analysis. Measurements were taken on a tattooed area and on non-tattooed area located on the symmetrically opposite side of the body.

Adhesive tattoos were placed on the volar forearm and a wet sponge was used to push firmly against the skin, then held around $30 \mathrm{~s}$. The skin was dried and finally, after 20 min wearing the tattoo, homeostasis parameters were measured.

To evaluate the moisturizer effect, the same amount of petrolatum was then applied on both measured areas (tattooed and non-tattooed skin) and after $30 \mathrm{~min}$, measurements were taken again. Antioxidant capacity was also only measured before petrolatum application.

Other variables: age, gender, phototype, past systemic or dermatological pathology, and medication, smoking and drinking habits were collected. Additionally, information was obtained about the tattoo, such as time of evolution, color, anatomical place, pathological reactions, subjective problems and cosmetic care.

\subsection{Statistical Analysis}

Descriptive statistics were used to present the characteristics of the sample. Continuous data were expressed as the mean and standard deviation. The absolute and relative frequency distributions were calculated for qualitative variable. The normality of the distribution for each parameter was checked with the Kolmogorov-Smirnov test.

To compare continuous data between tattooed skin and non-tattooed skin, the student's $t$-test for paired samples or the Wilcoxon signed-rank test were used depending on the normality of the distribution. The same procedure was carried out to compare data from moisturized and non-moisturized skin. To compare data from skin with permanent tattoos skin and adhesive tattoos, the student's $t$-test for independent samples or the MannWhitney $U$ test were used depending on the normality of the distribution. The Pearson correlation coefficient was calculated to test for possible correlation between continuous variables. Two-sided $p$-values $<0.05$ were considered as the level of statistical significance. SPSS version 24.0 (SPSS Inc, Chicago, IL, USA) was used for statistical analyzes.

\section{Results}

\subsection{Participants' Features}

This study included 67 samples (34 permanent and 33 adhesive tattoos) and 67 controls. The baseline characteristics of participants are given in Table 1.

Regarding permanent tattoos, $82.40 \%$ used black pigments only (28/34). Men had $52.90 \%$ of the tattoos (18/34). The mean age of participants in this group was 26.12 (SD 4.74) years old. The mean age of the tattoos was 24.62 (SD 24.33) months. The most frequent anatomical site for a tattoo was the upper limbs (19/34). Three past adverse events associated with tattoos were reported: one allergic reaction, and two itchiness associations.

Regarding adhesive tattoos, $54.50 \%$ were on males (18/33) and the mean age of the participants was 26.45 (SD 9.82) years old. All the adhesive tattoos were on the arm and had more than one color. 
Table 1. Descriptive characteristics of permanent and adhesive tattoos and the subjects they belong to.

\begin{tabular}{ccc}
\hline Sociodemographic Features & Permanent Tattoos $(n=34)$ & Adhesive Tattoos $(n=33)$ \\
\hline Age (years) $($ mean (range) $)$ & $26.12(21-41)$ & $26.45(19-53)$ \\
\hline Gender, $n(\%)$ & & \\
\hline Male & $18(52.9 \%)$ & $18(54.5 \%)$ \\
\hline Female & $16(47.1 \%)$ & $15(45.5 \%)$ \\
\hline Phototype, $n(\%)$ & & $0(0 \%)$ \\
\hline I & $1(2.9 \%)$ & $4(12.1 \%)$ \\
\hline II & $4(11.8 \%)$ & $25(75.8 \%)$ \\
\hline III & $23(67.6 \%)$ & $4(12.1 \%)$ \\
\hline IV & $6(17.6 \%)$ &
\end{tabular}

History of cutaneous pathology, $n$

(\%)

\begin{tabular}{|c|c|c|}
\hline Neoplasm & $2(5.9 \%)$ & $0(0 \%)$ \\
\hline Atopic dermatitis & $6(17.6 \%)$ & $6(18.2 \%)$ \\
\hline Other dermatitis & $2(5.9 \%)$ & $0(0 \%)$ \\
\hline \multicolumn{3}{|l|}{ Systemic medication, $n(\%)$} \\
\hline Contraceptives & $9(26.5 \%)$ & $8(24.2 \%)$ \\
\hline Other & $3(8.8 \%)$ & $3(9.1 \%)$ \\
\hline \multicolumn{3}{|l|}{ Topic medication, $n(\%)$} \\
\hline Corticoids & $5(14.7 \%)$ & $2(6.1 \%)$ \\
\hline Other & $0(0 \%)$ & $2(6.1 \%)$ \\
\hline \multicolumn{3}{|l|}{ Smokers } \\
\hline$n(\%)$ & $9(26.5 \%)$ & $2(6.1 \%)$ \\
\hline $\begin{array}{l}\text { Mean smoking pack per day } \\
\text { (range) }\end{array}$ & $1.31(0-25)$ & $0.24(0-4)$ \\
\hline \multicolumn{3}{|l|}{ Drinkers, $n(\%)$} \\
\hline$n(\%)$ & $19(55.9 \%)$ & $18(54.5 \%)$ \\
\hline Mean alcohol unit/day (range) & $0.27(0-1.14)$ & $0.25(0-1)$ \\
\hline $\begin{array}{l}\text { Tattoo duration (months) (mean } \\
\text { (range)) }\end{array}$ & $24.62(1-108)$ & - \\
\hline \multicolumn{3}{|l|}{ Pathologic reaction, $n(\%)$} \\
\hline Cutaneous allergy & $1(2.9 \%)$ & $0(0 \%)$ \\
\hline Itchiness & $2(5.9 \%)$ & $0(0 \%)$ \\
\hline Regular moisturizing, $n(\%)$ & $8(23.5 \%)$ & $0(0 \%)$ \\
\hline \multicolumn{3}{|l|}{ Localization, $n(\%)$} \\
\hline Head and neck & $3(8.8 \%)$ & $0(0 \%)$ \\
\hline Upper limbs & $19(55.9 \%)$ & $33(100 \%)$ \\
\hline Trunk & $8(23.5 \%)$ & $0(0 \%)$ \\
\hline Lower limbs & $4(11.8 \%)$ & $0(0 \%)$ \\
\hline \multicolumn{3}{|l|}{ Color, $n(\%)$} \\
\hline Black pigments only & $28(82.4 \%)$ & $0(0 \%)$ \\
\hline Other combinations & $6(17.6 \%)$ & $33(100 \%)$ \\
\hline
\end{tabular}

Data are expressed as relative (absolute) frequencies and means (standard deviation (SD). 


\subsection{Skin Homeostasis Analysis between Skin with Permanent Tattoos and Non-Tattooed Skin}

The skin homeostasis parameters in skin with permanent tattoos and non-tattooed skin are summarized in Table 2.

Table 2. Differences in biophysical parameters between skin with permanent tattoos and non-tattooed skin.

\begin{tabular}{|c|c|c|c|c|c|c|}
\hline & \multicolumn{3}{|c|}{ Before Moisturizer $(n=34)$} & \multicolumn{3}{|c|}{ After Moisturizer $(n=34)$} \\
\hline & $\begin{array}{c}\text { Skin with } \\
\text { Permanent Tattoos }\end{array}$ & $\begin{array}{l}\text { Non-Tattooed } \\
\text { Skin }\end{array}$ & $p$ & $\begin{array}{c}\text { Skin with } \\
\text { Permanent Tattoos }\end{array}$ & $\begin{array}{l}\text { Non-Tattooed } \\
\text { Skin }\end{array}$ & $p$ \\
\hline $\begin{array}{c}\text { Temperature } \\
\left({ }^{\circ} \mathrm{C}\right)\end{array}$ & $\begin{array}{c}30.47 \\
(1.46 \mathrm{SD})\end{array}$ & $\begin{array}{c}31.01 \\
(1.27 \mathrm{SD})\end{array}$ & $0.001 *$ & $\begin{array}{c}30.18 \\
(1.37 \mathrm{SD})\end{array}$ & $\begin{array}{c}30.61 \\
(1.32 \mathrm{SD})\end{array}$ & $<0.001^{* *}$ \\
\hline $\begin{array}{c}\text { TEWL } \\
\left(\mathrm{g} / \mathrm{h} / \mathrm{m}^{2}\right)\end{array}$ & $\begin{array}{c}8.72 \\
(5.42 \mathrm{SD})\end{array}$ & $\begin{array}{c}8.36 \\
(5.80 \mathrm{SD})\end{array}$ & 0.857 & $\begin{array}{c}7.90 \\
(4.03 \mathrm{SD})\end{array}$ & $\begin{array}{c}8.84 \\
(5.67 \mathrm{SD})\end{array}$ & 0.433 \\
\hline $\mathrm{SCH}(\mathrm{AU})$ & $\begin{array}{c}48.24 \\
(16.95 \mathrm{SD})\end{array}$ & $\begin{array}{c}44.15 \\
(15.26 \mathrm{SD})\end{array}$ & $0.008 *$ & $\begin{array}{c}44.32 \\
(13.05 \mathrm{SD})\end{array}$ & $\begin{array}{c}40.78 \\
(13.17 \mathrm{SD})\end{array}$ & $0.047^{* *}$ \\
\hline Erythema (AU) & $\begin{array}{c}96.77 \\
(141.43 \mathrm{SD}) \\
\end{array}$ & $\begin{array}{c}280.08 \\
(88.76 \mathrm{SD})\end{array}$ & $<0.001 *$ & $\begin{array}{c}98.33 \\
(142.78 \mathrm{SD})\end{array}$ & $\begin{array}{c}258.00 \\
(94.46 \mathrm{SD})\end{array}$ & $<0.001$ ** \\
\hline $\mathrm{Q} 1(\mathrm{uC})$ & $\begin{array}{c}0.97 \\
(0.46 \mathrm{SD})\end{array}$ & $\begin{array}{c}1.08 \\
(0.64 \mathrm{SD})\end{array}$ & 0.490 & - & - & - \\
\hline Q2 (uC) & $\begin{array}{c}4.44 \\
(1.36 \mathrm{SD})\end{array}$ & $\begin{array}{c}4.13 \\
(1.21 \mathrm{SD})\end{array}$ & 0.235 & - & - & - \\
\hline QT (uC) & $\begin{array}{c}5.40 \\
(1.71 \mathrm{SD})\end{array}$ & $\begin{array}{c}5.20 \\
(1.50 \mathrm{SD})\end{array}$ & 0.598 & - & - & - \\
\hline
\end{tabular}

AU, arbitrary units; $\mathrm{SCH}$, stratum corneum hydration; TEWL, transepidermal water loss; uC, microcoulombs; Q1, fast antioxidant capacity; Q2, slow antioxidant capacity; QT, total antioxidant capacity. The $p$ value after using Student $t$-test for paired samples or Wilcoxon signed-rank test where necessary, depending on the normality of the distribution, to compare biophysical parameters between skin with permanent tattoos and non-tattooed skin. * $p$ value after using Student's $t$ test for paired samples to compare skin with permanent tattoos and non-tattooed skin before moisturizer. ${ }^{* *} p$ value after using Student's $t$ test for paired samples to compare skin with permanent tattoos and non-tattooed skin after moisturizer.

Temperature was lower in tattooed skin than in non-tattooed skin $\left(30.47{ }^{\circ} \mathrm{C}\right.$ vs. $\left.31.01{ }^{\circ} \mathrm{C} ; p=0.001\right)$, while SCH (48.24 AU vs. $\left.44.15 \mathrm{AU} ; p=0.008\right)$ was higher in tattooed skin. The TEWL and TAC values did not differ between tattooed and non-tattooed skin before petrolatum application.

After petrolatum application, the temperature of tattooed skin was lower $\left(30.18^{\circ} \mathrm{C}\right.$ vs. $\left.30.61{ }^{\circ} \mathrm{C} ; p<0.001\right)$ than non-tattooed skin, while $\mathrm{SCH}$ was higher in tattooed skin (44.32 AU vs. 40.78 AU; $p=0.047$ ). No differences were found in TEWL values after moisturization.

\subsection{Skin Homeostasis Analysis between Skin with Adhesive Tattoos and Non-Tattooed Skin}

Skin homeostasis changes were found between skin with adhesive tattoos and nontattooed skin (Table 3).

Statistically significant differences were found in non-moisturized samples. Temperature $\left(30.78{ }^{\circ} \mathrm{C}\right.$ vs. $\left.32.05{ }^{\circ} \mathrm{C} ; p<0.001\right), \mathrm{SCH}(21.19 \mathrm{AU}$ vs. $41.31 \mathrm{AU} ; p<0.001)$ and TAC (1.27 microcoulombs (uC) vs. $3.48 \mathrm{uC} ; p<0.001)$ were lower in skin with adhesive tattoos skin, while TEWL $\left(8.65 \mathrm{~g} / \mathrm{h} / \mathrm{m}^{2}\right.$ vs. $\left.6.99 \mathrm{~g} / \mathrm{h} / \mathrm{m}^{2} ; p=0.003\right)$ was higher in skin with adhesive tattoos.

Similar results were obtained after the skin was moisturized, except for non-significant differences in TEWL. 
Table 3. Differences in biophysical parameters between skin with adhesive tattoos and non-tattooed skin.

\begin{tabular}{|c|c|c|c|c|c|c|}
\hline & \multicolumn{3}{|c|}{ Before Moisturizer $(n=33)$} & \multicolumn{3}{|c|}{ After Moisturizer $(n=33)$} \\
\hline & $\begin{array}{c}\text { Skin with Adhesive } \\
\text { Tattoos }\end{array}$ & $\begin{array}{l}\text { Non-Tattooed } \\
\text { Skin }\end{array}$ & $p$ & $\begin{array}{c}\text { Skin with Adhesive } \\
\text { Tattoos }\end{array}$ & $\begin{array}{c}\text { Non-Tattooed } \\
\text { Skin }\end{array}$ & $p$ \\
\hline Temperature $\left({ }^{\circ} \mathrm{C}\right)$ & $\begin{array}{c}30.78 \\
(1.11 \mathrm{SD})\end{array}$ & $\begin{array}{c}32.05 \\
(0.86 \mathrm{SD})\end{array}$ & $<0.001$ * & $\begin{array}{c}31.83 \\
(0.68 \mathrm{SD})\end{array}$ & $\begin{array}{c}32.32 \\
(0.66 \mathrm{SD})\end{array}$ & $<0.001$ ** \\
\hline TEWL $\left(\mathrm{g} / \mathrm{h} / \mathrm{m}^{2}\right)$ & $\begin{array}{c}8.65 \\
(3.11 \mathrm{SD}) \\
\end{array}$ & $\begin{array}{c}6.99 \\
(2.10 \mathrm{SD})\end{array}$ & $0.003 *$ & $\begin{array}{c}7.31 \\
(2.14 \mathrm{SD})\end{array}$ & $\begin{array}{c}7.88 \\
(1.63 \mathrm{SD})\end{array}$ & 0.062 \\
\hline $\mathrm{SCH}(\mathrm{AU})$ & $\begin{array}{c}21.19 \\
(6.71 \mathrm{SD})\end{array}$ & $\begin{array}{c}41.31 \\
(9.18 \mathrm{SD})\end{array}$ & $<0.001$ * & $\begin{array}{c}16.37 \\
(7.27 \mathrm{SD})\end{array}$ & $\begin{array}{c}31.45 \\
(9.99 \mathrm{SD})\end{array}$ & $<0.001$ ** \\
\hline Erythema (AU) & $\begin{array}{c}312.20 \\
(211.14 \mathrm{SD})\end{array}$ & $\begin{array}{c}212.61 \\
(37.01 \mathrm{SD})\end{array}$ & $0.021 *$ & $\begin{array}{c}335.34 \\
(225.93 \mathrm{SD}) \\
\end{array}$ & $\begin{array}{c}194.67 \\
(38.88 \mathrm{SD})\end{array}$ & $0.001 * *$ \\
\hline $\mathrm{Q} 1(\mathrm{uC})$ & $\begin{array}{c}0.17 \\
(0.17 \mathrm{SD}) \\
\end{array}$ & $\begin{array}{c}0.62 \\
(0.38 \mathrm{SD}) \\
\end{array}$ & $<0.001$ * & - & - & - \\
\hline Q2 (uC) & $\begin{array}{c}1.10 \\
(0.76 \mathrm{SD}) \\
\end{array}$ & $\begin{array}{c}2.86 \\
(1.18 \mathrm{SD}) \\
\end{array}$ & $<0.001$ * & - & - & - \\
\hline $\mathrm{QT}(\mathrm{uC})$ & $\begin{array}{c}1.27 \\
(0.91 \mathrm{SD})\end{array}$ & $\begin{array}{c}3.48 \\
(1.51 \mathrm{SD})\end{array}$ & $<0.001$ * & - & - & - \\
\hline
\end{tabular}

$\mathrm{AU}$, arbitrary units; $\mathrm{SCH}$, stratum corneum hydration; TEWL, transepidermal water loss; uC, microcoulombs; Q1, fast antioxidant capacity; Q2, slow antioxidant capacity; QT, total antioxidant capacity. The $p$ value after using Student $t$-test for paired samples or Wilcoxon signed-rank test where necessary, depending on the normality of the distribution, to compare biophysical parameters between skin with adhesive tattoos and non-tattooed skin. * $p$ value after using Student's $t$ test for paired samples to compare skin with adhesives tattoos and non-tattooed skin before moisturizer. ** $p$ value after using Student's $t$ test for paired samples to compare skin with adhesives tattoos and non-tattooed skin after moisturizer.

\subsection{Skin Homeostasis Analysis between Skin with Permanent Tattoos and Skin with Adhesive Tattoos}

Differences were found between skin with permanent tattoos and skin with adhesive tattoos (Table 4).

Table 4. Differences in biophysical parameters between skin with permanent tattoos and skin with adhesive tattoos.

\begin{tabular}{|c|c|c|c|c|c|c|}
\hline & \multicolumn{3}{|c|}{ Before Moisturizer } & \multicolumn{3}{|c|}{ After Moisturizer } \\
\hline & $\begin{array}{c}\text { Skin with } \\
\text { Permanent Tattoos } \\
(n=34)\end{array}$ & $\begin{array}{c}\text { Skin with Adhesive } \\
\text { Tattoos } \\
(n=33)\end{array}$ & $p$ & $\begin{array}{c}\text { Skin with } \\
\text { Permanent Tattoos } \\
(n=34)\end{array}$ & $\begin{array}{c}\text { Skin with Adhesive } \\
\text { Tattoos } \\
(n=33)\end{array}$ & $p$ \\
\hline Temperature $\left({ }^{\circ} \mathrm{C}\right)$ & $\begin{array}{c}30.47 \\
(1.46 \mathrm{SD})\end{array}$ & $\begin{array}{c}30.78 \\
(1.11 \mathrm{SD})\end{array}$ & 0.322 & $\begin{array}{c}30.18 \\
(1.37 \mathrm{SD})\end{array}$ & $\begin{array}{c}31.83 \\
(0.68 \mathrm{SD})\end{array}$ & $<0.001 * *$ \\
\hline TEWL $\left(\mathrm{g} / \mathrm{h} / \mathrm{m}^{2}\right)$ & $\begin{array}{c}8.72 \\
(5.42 \mathrm{SD})\end{array}$ & $\begin{array}{c}8.65 \\
(3.11 \mathrm{SD})\end{array}$ & 0.132 & $\begin{array}{c}7.90 \\
(4.03 \mathrm{SD})\end{array}$ & $\begin{array}{c}7.31 \\
(2.14 \mathrm{SD})\end{array}$ & 0.888 \\
\hline SCH (AU) & $\begin{array}{c}48.24 \\
(16.95 \mathrm{SD})\end{array}$ & $\begin{array}{c}21.19 \\
(6.71 \mathrm{SD})\end{array}$ & $<0.001 *$ & $\begin{array}{c}44.32 \\
(13.05 \mathrm{SD})\end{array}$ & $\begin{array}{c}16.37 \\
(7.27 \mathrm{SD})\end{array}$ & $<0.001 * *$ \\
\hline Erythema (AU) & 96.77 (141.43 SD) & $\begin{array}{c}312.20 \\
(211.14 \mathrm{SD})\end{array}$ & $<0.001 *$ & $\begin{array}{c}98.33 \\
(142.78 \text { SD) }\end{array}$ & $\begin{array}{c}335.34 \\
(225.93 \mathrm{SD})\end{array}$ & $<0.001 * *$ \\
\hline Q1 (uC) & $\begin{array}{c}0.97 \\
(0.46 \mathrm{SD})\end{array}$ & $\begin{array}{c}0.17 \\
(0.17 \mathrm{SD})\end{array}$ & $<0.001 *$ & - & - & - \\
\hline $\mathrm{Q} 2(\mathrm{uC})$ & $\begin{array}{c}4.44 \\
(1.36 \mathrm{SD})\end{array}$ & $\begin{array}{c}1.10 \\
(0.76 \mathrm{SD})\end{array}$ & $<0.001 *$ & - & - & - \\
\hline QT (uC) & $\begin{array}{c}5.40 \\
(1.71 \mathrm{SD})\end{array}$ & $\begin{array}{c}1.27 \\
(0.91 \mathrm{SD})\end{array}$ & $<0.001 *$ & - & - & - \\
\hline
\end{tabular}

AU, arbitrary units; SCH, stratum corneum hydration; TEWL, transepidermal water loss; uC, microcoulombs; Q1, fast antioxidant capacity; Q2, slow antioxidant capacity; QT, total antioxidant capacity. The $p$ value after using Student's t-test for independent samples or Mann-Whitney U test where necessary, depending on the normality of the distribution, to compare biophysical parameters between permanent tattooed skin and adhesive tattooed skin. ${ }^{*} p$ value after using Student's $t$ test for independent samples to compare skin with permanent tattoos and skin with adhesive tattoos before moisturizer. ${ }^{* *} p$ value after using Student's $t$ test for independent samples to compare skin with permanent tattoos and skin with adhesive tattoos after moisturizer. 
Skin with permanent tattoos presented higher SCH (48.24 AU vs. 21.19 AU; $p<0.001)$ and TAC ( $5.40 \mathrm{uC}$ vs. $1.27 \mathrm{uC} ; p<0.001)$ values before the petrolatum application. Similar TEWL values were observed in skin with permanent and adhesive tattoos.

\subsection{Skin Homeostasis Analysis Comparing before and after Petrolatum Application}

Changes were observed between non-tattooed skin, skin with permanent tattoos and skin with adhesive tattoos after petrolatum application (Table 5).

Table 5. Differences in biophysical parameters between moisturized skin and non-moisturized skin for non-tattooed skin, skin with permanent tattoos and skin with adhesive tattoos.

\begin{tabular}{|c|c|c|c|c|c|c|c|c|c|}
\hline & \multicolumn{3}{|c|}{ Non-Tattooed Skin $(n=34)$} & \multicolumn{3}{|c|}{ Skin with Permanent Tattoos $(n=34)$} & \multicolumn{3}{|c|}{ Skin with Adhesive Tattoos $(n=33)$} \\
\hline & $\begin{array}{c}\text { After } \\
\text { Moisturization }\end{array}$ & $\begin{array}{c}\text { Mean Difference } \\
\text { between before } \\
\text { and after } \\
\text { Moisturization }\end{array}$ & $\begin{array}{c}p \text { Value } \\
\text { Comparing } \\
\text { before vs. after } \\
\text { Moisturization }\end{array}$ & $\begin{array}{c}\text { After } \\
\text { Moisturization }\end{array}$ & $\begin{array}{c}\text { Mean Difference } \\
\text { between before } \\
\text { and after } \\
\text { Moisturization }\end{array}$ & $\begin{array}{c}p \text { Value } \\
\text { Comparing } \\
\text { before vs. after } \\
\text { Moisturization }\end{array}$ & $\begin{array}{c}\text { After } \\
\text { Moisturization }\end{array}$ & $\begin{array}{c}\text { Mean Difference } \\
\text { between before } \\
\text { and after } \\
\text { Moisturization }\end{array}$ & $\begin{array}{c}p \text { Value } \\
\text { Comparing } \\
\text { before vs. after } \\
\text { Moisturization }\end{array}$ \\
\hline Temperature $\left({ }^{\circ} \mathrm{C}\right)$ & $\begin{array}{c}30.61 \\
(1.32 \mathrm{SD})\end{array}$ & $\begin{array}{c}-0.36 \\
(0.73 \mathrm{SD}) \\
\end{array}$ & $0.008 *$ & $\begin{array}{c}30.18 \\
(1.37 \mathrm{SD})\end{array}$ & $\begin{array}{c}-0.26 \\
(0.74 \mathrm{SD}) \\
\end{array}$ & 0.050 ** & $\begin{array}{c}31.83 \\
(0.68 \mathrm{SD})\end{array}$ & $\begin{array}{c}+1.05 \\
(0.89 \mathrm{SD}) \\
\end{array}$ & $<0.001 * * *$ \\
\hline TEWL $\left(\mathrm{g} / \mathrm{h} / \mathrm{m}^{2}\right)$ & $\begin{array}{c}8.84 \\
\text { (5.67 SD) } \\
\end{array}$ & $\begin{array}{c}+0.33 \\
(6.88 \mathrm{SD}) \\
\end{array}$ & 0.268 & $\begin{array}{c}7.90 \\
(4.03 \mathrm{SD}) \\
\end{array}$ & $\begin{array}{c}-0.83 \\
(6.32 \mathrm{SD})\end{array}$ & 0.903 & $\begin{array}{c}7.31 \\
(2.14 \mathrm{SD})\end{array}$ & $\begin{array}{c}-1.35 \\
(3.28 \mathrm{SD}) \\
\end{array}$ & $0.025 * * *$ \\
\hline $\mathrm{SCH}(\mathrm{AU})$ & $\begin{array}{c}40.78 \\
(13.17 \mathrm{SD})\end{array}$ & $\begin{array}{c}-4.01 \\
(17.49 \mathrm{SD}) \\
\end{array}$ & 0.197 & $\begin{array}{c}44.32 \\
(13.05 \mathrm{SD}) \\
\end{array}$ & $\begin{array}{c}-4.26 \\
(18.15 \mathrm{SD}) \\
\end{array}$ & 0.187 & $\begin{array}{c}16.37 \\
(7.27 \mathrm{SD})\end{array}$ & $\begin{array}{c}-4.83 \\
(5.00 \mathrm{SD}) \\
\end{array}$ & $<0.001 * * *$ \\
\hline Erythema (AU) & $\begin{array}{c}258.85 \\
\text { (94.46 SD) }\end{array}$ & $\begin{array}{c}-21.56 \\
(30.16 \mathrm{SD})\end{array}$ & $<0.001$ * & $\begin{array}{c}98.33 \\
(142.78 \mathrm{SD})\end{array}$ & $\begin{array}{c}+2.29 \\
(24.76 \mathrm{SD})\end{array}$ & 0.875 & $\begin{array}{c}335.34 \\
\text { (225.93 SD) }\end{array}$ & $\begin{array}{c}+23.14 \\
(143.93 \mathrm{SD})\end{array}$ & 0.396 \\
\hline
\end{tabular}

AU, arbitrary units; SCH, stratum corneum hydration; TEWL, transepidermal water loss. The $p$ value after using Student's $t$-test for paired samples or Wilcoxon signed-rank test where necessary, depending on the normality of the distribution, to compare biophysical parameters between moisturized skin and non-moisturized skin in skin with permanent tattoos, adhesive tattoos and non-tattooed skin. ${ }^{*} p$ value after using Student's $t$ test for paired samples to compare skin homeostasis parameters on non-tattooed skin before and after moisturization. ** $p$ value after using Student's $t$ test for paired samples to compare skin homeostasis parameters on skin with permanent tattoos before and after moisturization. ${ }^{* * *} p$ value after using Student's $t$ test for paired samples to compare skin homeostasis parameters on skin with adhesive tattoos before and after moisturization.

The temperature decreased by $-0.36(\mathrm{SD} 0.73)^{\circ} \mathrm{C}$ in non-tattooed skin $(p=0.008)$ and $-0.26(\mathrm{SD} 0.74){ }^{\circ} \mathrm{C}$ in skin with permanent tattoos $(p=0.050)$ after petrolatum application. No differences in SCH and TEWL were observed after applying petrolatum to skin with permanent tattoos or non-tattooed skin.

After applying petrolatum to skin with adhesive tattoos, the temperature increased by $1.0{ }^{\circ} \mathrm{C}$ (SD 0.89) $(p<0.001)$, TEWL decreased by -1.35 (SD 3.28) $\mathrm{g} / \mathrm{h} / \mathrm{m}^{2}(p=0.025)$ and SCH decreased by -4.83 (SD 5.00) AU ( $p<0.001$ ).

The effects of petrolatum on erythema were different in non-tattooed skin and tattooed skin. Erythema decreased by -21.56 (SD 5.25) AU in non-tattooed skin $(p<0.001)$, while no effect was observed in skin with permanent tattoos $(p=0.875)$ or skin with adhesive tattoos $(p=0.396)$.

\section{Discussion}

Permanent tattoos do not significantly affect skin homeostasis compared to nontattooed skin. Only temperature and erythema decreases were observed. Temporary adhesive tattoos alter cutaneous homeostasis, as they reduce temperature, $\mathrm{SCH}$ and antioxidant capacity, and increase TEWL, compared to non-tattooed skin. Petrolatum reduces TEWL with adhesive tattoos.

To the best of our knowledge, only one previous study has compared skin homeostasis parameters between permanent tattoos and non-tattooed skin [17]. Norrelet et al. found no differences in TEWL and $\mathrm{pH}$ values between non-tattooed and tattooed skin in 28 tattoos. Similarly, they found higher capacitance on tattooed skin [17], in agreement with the higher $\mathrm{SCH}$ values on tattooed skin observed in our study. Skin barrier disruption is observed after needle injury micro-traumatisms, which is shown by the slight increases in TEWL observed in our report. As it has been previously shown, this increased water flow may indirectly lead to increased hydration [19].

There are no studies regarding the epidermal barrier function of skin with adhesive tattoos. Increased TEWL values are found after the application and removal of adhesives, such as wound dressing, resulting in skin barrier impairment [20]. Our study showed 
higher TEWL in skin with adhesive tattoos, associated with skin damage. SCH and TAC were also altered in skin with adhesive tattoos. Since high TEWL and low SCH values indicate damage to the skin barrier function [21], and adhesive modifies these parameters, adhesive tattoos may negatively affect the skin. In that way, people suffering from cutaneous disease with a skin barrier dysfunction, such as psoriasis or atopic dermatitis [22,23], should avoid repeated use of adhesive tattoos. Moreover, these findings should be considered in the development of new electronic adhesive devices for medical use, which could also damage the skin [24]. Future studies on this matter may determine if skin properties are affected by the use of adhesive medical devices.

Skin with adhesive tattoos showed lower antioxidant values compared to non-tattooed skin. Previously, it has also been observed that tattoo exposure induces mild oxidative stress and enhances the activity of certain antioxidants [25]. The composition of adhesive tattoos is usually unknown, but they usually contain colorants and hazardous chemicals, such as phthalate ester, aromatic amines, heavy metals or even adhesive substances [16], which may lead to skin irritation, releasing proinflammatory cytokines and inducing oxidative stress [26], which may explain the decreased antioxidant capacity of adhesive tattooed skin.

Petrolatum was used to assess if tattooed skin reacts similarly to non-tattooed skin after moisturizing. Petrolatum is known to reduce TEWL on healthy skin [27]. Our study did not show significant differences for TEWL in moisturized and non-moisturized skin after a single application, which is consistent with previous reports [28]. The petrolatum effect in improving TEWL might be observed for long-term application, which involves creating a water-repellent lipid layer on the skin surface that blocks water loss [29]. However, a significant decrease in TEWL after a single petrolatum application was observed in adhesive tattooed skin. Petrolatum has been proven to help to reduce erythema following skin damage induced by different agents, such as ultraviolet radiation or topical medication $[30,31]$. Petrolatum is a hypoallergenic substance that does not bind to proteins or undergo chemical alteration in the skin [32].

This study was subject to several limitations: a limited sample size and a short followup period. Future research could measure the skin barrier function parameters of other types of temporary tattoos, such as henna or jagua, which have been reported to cause skin pathologies, especially allergic reactions [33,34].

\section{Conclusions}

Permanent tattoos do not significantly affect skin barrier function. Adhesive tattoos cause skin barrier dysfunction, showing increased TEWL and decreased SCH values, as well as increased temperature and decreased antioxidant skin capacity. Tattooed and non-tattooed skin respond in different ways to moisturizers.

Author Contributions: Conceptualization, J.-P.S.-S., S.A.-S. and T.M.-V.; methodology, S.A.-S. and T.M.-V.; software, A.B.-E.; validation, S.A.-S. and T.M.-V.; formal analysis, J.-P.S.-S., S.A.-S. and T.M.-V.; investigation, J.-P.S.-S., S.A.-S. and T.M.-V.; resources, A.B.-E., J.-P.S.-S., S.A.-S. and T.M.-V.; data curation, J.-P.S.-S.; writing-original draft preparation, J.-P.S.-S.; writing-review and editing, S.A.-S. and T.M.-V.; visualization, S.A.-S. and T.M.-V.; supervision, S.A.-S. and T.M.-V.; project administration, none; funding acquisition, none. All authors have read and agreed to the published version of the manuscript.

Funding: This research received no external funding.

Institutional Review Board Statement: The study was conducted according to the guidelines of the Declaration of Helsinki, and approved by the Ethics Committee of Hospital Universitario Virgen de las Nieves (protocol code HC01 and date of approval 18/02/2020).

Informed Consent Statement: Informed consent was obtained from all subjects involved in the study. Written informed consent has been obtained from the patients to publish this paper.

Data Availability Statement: The data presented in this study are available on request from the corresponding author. 
Acknowledgments: We would like to thank all the individuals who generously shared their time to participate in this research. We would like to thank Charlotte Bower, for improving the English of this manuscript.

Conflicts of Interest: The authors declare no conflict of interest.

\section{References}

1. Wohlrab, S.; Stahl, J.; Kappeler, P.M. Modifying the body: Motivations for getting tattooed and pierced. Body Image 2007, 4, 87-95. [CrossRef]

2. Kluger, N.; Seité, S.; Taieb, C. The prevalence of tattooing and motivations in five major countries over the world. J. Eur. Acad. Dermatol. Venereol. 2019, 33, e484-e486. [CrossRef] [PubMed]

3. Borkenhagen, A.; Mirastschijski, U.; Petrowski, K.; Brähler, E. Tattoos in Germany: Prevalence, demographics, and health orientation. Bundesgesundheitsblatt Gesundh. Gesundh. 2019, 62, 1077-1082. [CrossRef]

4. Kluger, N.; Misery, L.; Seité, S.; Taieb, C. Tattooing: A national survey in the general population of France. J. Am. Acad. Dermatol. 2019, 81, 607-610. [CrossRef]

5. Kluger, N. Epidemiology of tattoos in industrialized countries. Tattooed Skin Health 2015, 48, 6-20.

6. Luetkemeier, M.J.; Hanisko, J.M.; Aho, K.M. Skin Tattoos Alter Sweat Rate and Na+ Concentration. Med. Sci. Sports Exerc. 2017, 49, 1432-1436. [CrossRef]

7. Laux, P.; Tralau, T.; Tentschert, J.; Blume, A.; Al Dahouk, S.; Bäumler, W.; Bernstein, E.; Bocca, B.; Alimonti, A.; Colebrook, H.; et al. A medical-toxicological view of tattooing. Lancet 2016, 387, 395-402. [CrossRef]

8. Sperry, K. Part I: History and methodology. Tattoos and tattooing. Am. J. Forensic. Med. Pathol. 1991, 12, 313-319. [CrossRef]

9. Sperry, K. Tattoos and tattooing. Part II: Gross pathology, histopathology, medical complications, and applications. Am. J. Forensic. Med. Pathol. 1992, 13, 7-17. [CrossRef]

10. Blasco-Morente, G.; Pérez-López, I.; Martínez-López, A.; Aneiros-Fernández, J.; Tercedor-Sánchez, J.; Arias-Santiago, S. Pigmented lymph nodes in a patient with melanoma: Tattoos. J. Eur. Acad. Dermatol. Venereol. 2015, 30, e116-e117. [CrossRef] [PubMed]

11. Sepehri, M.; Sejersen, T.; Qvortrup, K.; Lerche, C.M.; Serup, J. Tattoo Pigments Are Observed in the Kupffer Cells of the Liver Indicating Blood-Borne Distribution of Tattoo Ink. Dermatology 2017, 233, 86-93. [CrossRef]

12. Kazandjieva, J.; Tsankov, N. Tattoos: Dermatological complications. Clin. Dermatol. 2007, 25, 375-382. [CrossRef]

13. Kluger, N.; Koljonen, V. Tattoos, inks, and cancer. Lancet Oncol. 2012, 13, e161-e168. [CrossRef]

14. Navrazhina, K.; Goldman, B.; Leger, M.C. Atypical Intraepidermal Melanocytic Proliferation Masked by a Tattoo: Implications for Tattoo Artists and Public Health Campaigns. Cureus 2018, 10, e2975. [CrossRef]

15. Morse, D.; Campana, J.; Biggar, R.J.; Haughie, G.E.V.B.J. Letter: Reactions to adhesive tattoos in children. N. Engl. J. Med. 1975, 293, 153.

16. Rubio, L.; Guerra, E.; Garcia-Jares, C.; Lores, M. Body-decorating products: Ingredients of permanent and temporary tattoos from analytical and european regulatory perspectives. Anal. Chim. Acta 2019, 1079, 59-72. [CrossRef]

17. Nørreslet, L.B.; Serup, J.; Kezic, S.; Engebretsen, K.A.; Thyssen, J.P.; Agner, T.; Clausen, M.-L. Tattoos and skin barrier function: Measurements of TEWL, stratum corneum conductance and capacitance, pH, and filaggrin. Ski. Res. Technol. 2019, 25, 382-388. [CrossRef]

18. e-BQC I New Device for Antioxidant Capacity Determination [Internet]. Available online: http://e-bqc.com/ (accessed on 15 April 2020).

19. Suetake, T.; Sasai, S.; Zhen, Y.X.; Ohi, T.; Tagami, H. Functional analyses of the stratum corneum in scars. Sequential studies after injury and comparison among keloids, hypertrophic scars, and atrophic scars. Arch. Dermatol. 1996, 132, 1453-1458. [CrossRef]

20. Waring, M.; Bielfeldt, S.; Mätzold, K.; Wilhelm, K.; Butcher, M. An evaluation of the skin stripping of wound dressing adhesives. J. Wound Care 2011, 20, 412-422. [CrossRef] [PubMed]

21. Montero-Vilchez, T.; Segura-Fernández-Nogueras, M.-V.; Pérez-Rodríguez, I.; Soler-Gongora, M.; Martinez-Lopez, A.; FernándezGonzález, A.; Molina-Leyva, A.; Arias-Santiago, S. Skin Barrier Function in Psoriasis and Atopic Dermatitis: Transepidermal Water Loss and Temperature as Useful Tools to Assess Disease Severity. J. Clin. Med. 2021, 10, 359. [CrossRef]

22. Horimukai, K.; Morita, K.; Narita, M.; Kondo, M.; Kabashima, S.; Inoue, E.; Sasaki, T.; Niizeki, H.; Saito, H.; Matsumoto, K.; et al. Transepidermal water loss measurement during infancy can predict the subsequent development of atopic dermatitis regardless of filaggrin mutations. Allergol. Int. 2016, 65, 103-108. [CrossRef]

23. Montero-Vilchez, T.; Soler-Góngora, M.; Martínez-López, A.; Fernández-González, A.; Buendía-Eisman, A.; Molina-Leyva, A.; Arias-Santiago, S. Epidermal barrier changes in patients with psoriasis: The role of phototherapy. Photodermatol. Photoimmunol. Photomed. 2020, online ahead of print. [CrossRef]

24. Alberto, J.; Leal, C.; Fernandes, C.; Lopes, P.A.; Paisana, H.; De Almeida, A.T.; Tavakoli, M. Fully Untethered Battery-free Biomonitoring Electronic Tattoo with Wireless Energy Harvesting. Sci. Rep. 2020, 10, 1-11. [CrossRef]

25. Kubrak, O.I.; Atamaniuk, T.M.; Husak, V.V.; Drohomyretska, I.Z.; Storey, J.M.; Storey, K.B.; Lushchak, V.I. Oxidative stress responses in blood and gills of Carassius auratus exposed to the mancozeb-containing carbamate fungicide Tattoo. Ecotoxicol. Environ. Saf. 2012, 85, 37-43. [CrossRef] [PubMed]

26. Furue, M. Regulation of Filaggrin, Loricrin, and Involucrin by IL-4, IL-13, IL-17A, IL-22, AHR, and NRF2: Pathogenic Implications in Atopic Dermatitis. Int. J. Mol. Sci. 2020, 21, 5382. [CrossRef] [PubMed] 
27. Draelos, Z.D. The science behind skin care: Moisturizers. J. Cosmet. Dermatol. 2018, 17, 138-144. [CrossRef]

28. Samadi, A.; Nasrollahi, S.A.; Ashtiani, M.M.; Abels, C.; Firooz, A. Changes in skin barrier function following single and repeated applications of 4 types of moisturizers: A randomized controlled trial. J. Eur. Acad. Dermatol. Venereol. 2020, 34. [CrossRef] [PubMed]

29. Elias, P.M.; Wakefield, J.S.; Man, M.-Q. Moisturizers versus Current and Next-Generation Barrier Repair Therapy for the Management of Atopic Dermatitis. Skin Pharmacol. Physiol. 2019, 32, 1-7. [CrossRef] [PubMed]

30. Maarouf, M.; Kromenacker, B.W.; Brucks, E.S.; Hendricks, A.; Shi, V.Y. Reducing unpleasant side effects of topical 5-Fluorouracil treatment for actinic keratosis: A randomized controlled trial. J. Dermatol. Treat. 2019, 31, 175-179. [CrossRef]

31. Schleider, N.R.; Moskowitz, R.S.; Cort, D.H.; Horwitz, S.N.; Frost, P. Effects of emollients on ultraviolet-radiation-induced erythema of the skin. Arch. Dermatol. 1979, 115, 1188-1191. [CrossRef] [PubMed]

32. Soltanipoor, M.; Stilla, T.; Riethmüller, C.; Thyssen, J.P.; Sluiter, J.K.; Rustemeyer, T.; Fischer, T.W.; Kezic, S.; Angelova-Fischer, I. Specific barrier response profiles after experimentally induced skin irritation in vivo. Contact Dermat. 2018, 79, 59-66. [CrossRef]

33. de Groot, A.C. Side-effects of henna and semi-permanent 'black henna' tattoos: A full review. Contact Dermat. 2013, 69, 1-25. [CrossRef]

34. Bircher, A.J.; Hofmeier, K.S.; Schlegel, U.; Hauri, U. Genipin in Temporary Jagua Tattoos—Black Dye Causing Severe Allergic Dermatitis. Dermatitis 2019, 30, 375-376. [CrossRef] 